\title{
Prevalence of co-morbidities in a specialist weight management programme prior to bariatric surgery
}

\author{
DANIEL FOUNTAIN, ${ }^{1}$ MOHAMMED ALKHARAIJI, ${ }^{1}$ SHERIF AWAD, ${ }^{1,2}$ DAVID HUGHES, ${ }^{2}$ ISKANDAR IDRIS ${ }^{1,2}$
}

\begin{abstract}
Objective: There is limited evidence on the prevalence of patients' obesity-related co-morbidities and the effectiveness of specialist multidisciplinary weight management (Tier 3) programmes prior to bariatric surgery. We therefore evaluate and report the prevalence of co-morbidities in patients attending a Tier 3 service within the National Health Service. Methods: This was a prospective observational study of consecutive patients who attended the Tier 3 service at the East Midlands Bariatric Metabolic Institute throughout 2017.

Results: $\mathbf{4 3 0}$ patients attended the service over the study observation period. Twelve patients $(2.8 \%)$ were excluded from our analysis due to incomplete data. $70.8 \%$ of patients were women, mean age at baseline was 46.4 years, mean \pm SD weight and body mass index at baseline were $137.8 \pm 29.2 \mathrm{~kg}$ and $48.0 \pm 8.6 \mathrm{~kg} / \mathrm{m}^{2}$, respectively. The most common co-morbidities recorded at baseline were type 2 diabetes mellitus (31.1\%), hypertension (31.1\%), depression $(26.1 \%)$, obstructive sleep apnoea (23.2\%) and osteoarthritis $(15.6 \%)$. Significant weight loss was observed at the 3-month and 6-month follow-up points, but not at the 9- or 12-month follow-up points. $22.5 \%$ of patients achieved weight loss of $\geq 5 \%$.

Conclusion: The prevalence of co-morbidities within this Tier 3 service was high. While specialised weight management services may achieve moderate weight loss through a multidisciplinary intervention, future evaluation of clinical outcomes of specialist weight services should also include co-morbidity outcomes.

Br J Diabetes 2019;19:8-13
\end{abstract}

Key words: Tier 3 service, bariatric surgery, multidisciplinary, obesity-related co-morbidities

Division of Medical Sciences and Graduate Entry Medicine, School of Medicine, University of Nottingham, UK

2 East Midlands Bariatric Metabolic Institute, University Hospitals of Derby and Burton NHS Foundation Trust, Derby, UK

Address for correspondence: $\mathrm{Dr}$ Iskandar Idris

Division of Medical Sciences and Graduate Entry Medicine, School of Medicine, University of Nottingham, DE22 3DT, UK

E-mail: iskandar.idris@nottingham.ac.uk

https://doi.org/10.15277/bjd.2019.205

\section{Introduction}

Obesity is recognised as one of the leading healthcare challenges in the UK and globally. ${ }^{1}$ National Health Service (NHS) statistics showed that, in $2015,27 \%$ of UK adults were obese - an increase of $12 \%$ from 1993. ${ }^{2}$ The percentage of adults classified as obese (body mass index (BMI) $\geq 30 \mathrm{~kg} / \mathrm{m}^{2}$ ) has since remained steady, but there has been an alarming $18 \%$ increase in hospital admissions for obesity-related co-morbidities, rising from 525,000 in $2015 / 16$ to 617,000 in $2017 / 18 .^{2}$ Worryingly also, $20 \%$ of year 6 children (aged 10-11) and 10\% of reception year children (aged 4-5) are now classed as obese. ${ }^{2}$ Studies have shown that obesity increases the likelihood of developing serious co-morbidities including - but not limited to - diabetes, hypertension, ${ }^{3}$ gallstones, ${ }^{4}$ osteoarthritis, ${ }^{5}$ cardiovascular disease, ${ }^{6}$ sleep apnoea (among males) ${ }^{7}$ and fatty liver disease. ${ }^{8}$ Moderate sustained weight loss of $5-10 \%$ has been shown to be associated with significant clinical benefits in individuals with obesity and is therefore considered an important treatment goal. ${ }^{9}$

The UK NHS has recommended a Tiered structured model of weight management. ${ }^{10}$ Tier 1 interventions encompass general guidance and advice in the community as well as populationwide measures. Tier 2 interventions include more involved measures such as weight loss programmes, which are provided by local public health bodies or commercial providers (eg, Weight Watchers $^{\mathrm{TM}}$ and Slimming World ${ }^{\mathrm{TM}}$ ). Tier 3 interventions represent a specialist multidisciplinary team (MDT) managing individuals with obesity either in a community or hospital setting, comprising a minimum of a specialist dietician, physician and clinical psychologist. These services are typically funded by local health clinical commissioning groups. An important remit of a Tier 3 service is to prepare appropriately selected patients for bariatric surgical intervention (Tier 4). However, while the prevalence of co-morbidities in various community-based Tier 3 programmes has been reported, ${ }^{11}$ the prevalence of obesityrelated co-morbidities within hospital-based Tier 3 programmes is currently unclear.

This prospective observational study aimed to examine the prevalence of obesity-related co-morbidities and evidence for the effectiveness of Tier 3 interventions in patients admitted to the Tier 3 weight management service at East Midlands Bariatric Metabolic Institute (EMBMI) based at Derby and Burton Teaching Hospitals NHS Foundation Trust. The service was evaluated with the aid of the Standard Evaluation Framework for Weight 
Management Interventions ${ }^{12}$ and measured against the requirements stipulated in the Clinical Commissioning Policy: Complex and Specialised Obesity Surgery. ${ }^{10}$

\section{Methods \\ Setting}

EMBMI is a regional tertiary referral centre for bariatric and metabolic surgery which provides both Tier 3 and Tier 4 services. The Tier 3 service was developed in 2014 in response to the NHS England requirement in 2013 which mandated patients to access specialist Tier 3 interventions prior to undergoing bariatric surgery. This is a multicomponent specialist weight management service available to patients aged 18 years and over with complex obesity (defined as per the National Institute for Health and Care Excellence (NICE) guidelines as BMI $\geq 35 \mathrm{~kg} / \mathrm{m}^{2}$ with obesityrelated co-morbidities or $\mathrm{BMI} \geq 40 \mathrm{~kg} / \mathrm{m}^{2}$ without co-morbidities). This relates to the obesity class 2 and class 3 threshold for 'severely obese' and 'very severely obese'. Patients are referred by their general practitioner or hospital doctor for consideration of bariatric surgery. This was a time-limited multidisciplinary specialist weight intervention service with input from physicians to screen and manage patients' co-morbidities; specialist dieticians to educate patients on mindful eating, devise a structured framework for caloric restriction/eating behaviour (eg, portion control, slowing speed of eating and appropriate food choices); and a psychologist to screen and manage relevant mental health disorders. The intensity and frequency of follow-up visits were determined by patients' clinical need, guided by regular MDT meetings. Suitability for surgery was assessed at a minimum of 3 months from the first visit.

\section{Data collection}

Patients' data were obtained from electronic patient records. Inclusion criteria into this study were all patients who had a scheduled and attended appointment at any time during 2017. Baseline data were derived from the first Tier 3 appointment in 2017, from correspondence sent to the patient's GP and/or from clinical notes made during the appointment. The following data were collected: age, sex, race, date of first visit, weight, height, BMI, blood pressure, previous bariatric surgery (if applicable), Epworth score if symptomatic of sleep apnoea, endoscopy results, full blood count, urea and electrolytes, liver function, calcium, lipid profile, $\mathrm{HbA}_{1 \mathrm{c}}$, thyroid, details of co-morbidities present and relevant medications received. The follow-up data, however, only included weight and BMI. Short-term weight outcomes were also reported as mean weight change and 5\% weight loss of those completing the programme, as a means to compare with other programmes. Follow-up data were collected from a broader range of appointments including dietician appointments and education sessions. The follow-up data included only an updated weight and BMI. Percentage weight change was calculated as well as BMI change. Follow-up was arranged into groups of 3, 6, 9 and 12 months. Drop-outs were patients who did not attend follow-up appointments within Tier 3.

\section{Statistical analysis}

All referrals in 2017 were followed up until they completed or left the programme. Data were censured at 1 April 2018 to ensure full data were available. The baseline weight data were found to be not normally distributed via visual inspection of the histogram as well as the Shapiro-Wilk test. Therefore, the Wilcoxon signed ranks test was used to test the significance of the data. For missing data, the method of last observation carried forward was used. The criteria for statistical significance was set at $p \leq 0.05$. SPSS version 24.0.2 for Windows (SPSS Inc, Chicago, IL, USA) was used for any statistical analysis of the data. Descriptive patient characteristics were described as mean \pm standard deviation (SD).

\section{Results}

\section{Patient flow}

Data were collected from 418 patients as part of the evaluation study. Ninety-eight patients had follow-up data at 3 months, 94 had follow-up data at 6 months and 41 had follow-up data at 9 months, after which most patients were either discharged or referred for bariatric surgery. In total, 191 patients (45.69\%) had at least one point of follow-up data. Ninety-three patients who did not have follow-up at 3 months had follow-up at a further point during their treatment at the Tier 3 clinic. Figure 1 shows the full flow of patients through the Tier 3 service from admission to 12 months.

\section{Descriptive baseline patients' characteristics and co-morbidities}

A total of 430 patients were recorded as presenting to the Tier 3 clinic in 2017. Of these, 418 met the inclusion criteria for the evaluation study; the remaining 12 were excluded because there were no notes recorded for their visit or because vital information such as baseline weight was unavailable. Of those who met the inclusion criteria, 296 (70.8\%) were female and 122 (29.2\%) were male. The mean \pm SD baseline weight for the sample was $137.8 \pm 29.2 \mathrm{~kg}$ and the mean baseline BMI was $48.0 \pm 8.6 \mathrm{~kg} / \mathrm{m}^{2}$. The mean \pm SD age, weight and BMI for male patients was $47.0 \pm 12$ years, $154.2 \pm 32.7 \mathrm{~kg}$ and $48.1 \pm 8.8 \mathrm{~kg} / \mathrm{m}^{2}$, respectively. The respective figures for female patients were $45.8 \pm 11.8$ years, $131.1 \pm 32.7 \mathrm{~kg}$ and $48.0 \pm 8.8 \mathrm{~kg} / \mathrm{m}^{2}$. Overall, four patients (1.0\%) were classed as overweight, $12(2.9 \%)$ were class I obese, 48 (11.5\%) were class II obese and the remaining 354 $(84.7 \%)$ were class III obese. 267 (63.9\%) patients were identified as White British and a further 115 (27.5\%) had either not stated or not recorded ethnicity. The remaining 36 (8.6\%) patients were split between a variety of ethnicities including Bangladeshi, Black Caribbean and Mixed Other. Table 1 summarises the baseline characteristics of the sample. Ninety-eight patients had follow-up data at 3 months, 94 had follow-up data at 6 months, 41 had follow-up data at 9 months and 17 had follow-up at 12 months. In total, 191 (45.69\%) patients had at least one point of follow-up data. A total of 93 patients who did not have follow-up at 3 months had follow-up at a further point during their treatment at the Tier 3 clinic. 
Figure 1. Flowchart showing the progression of patients through the Tier 3 service from referral to 12 months

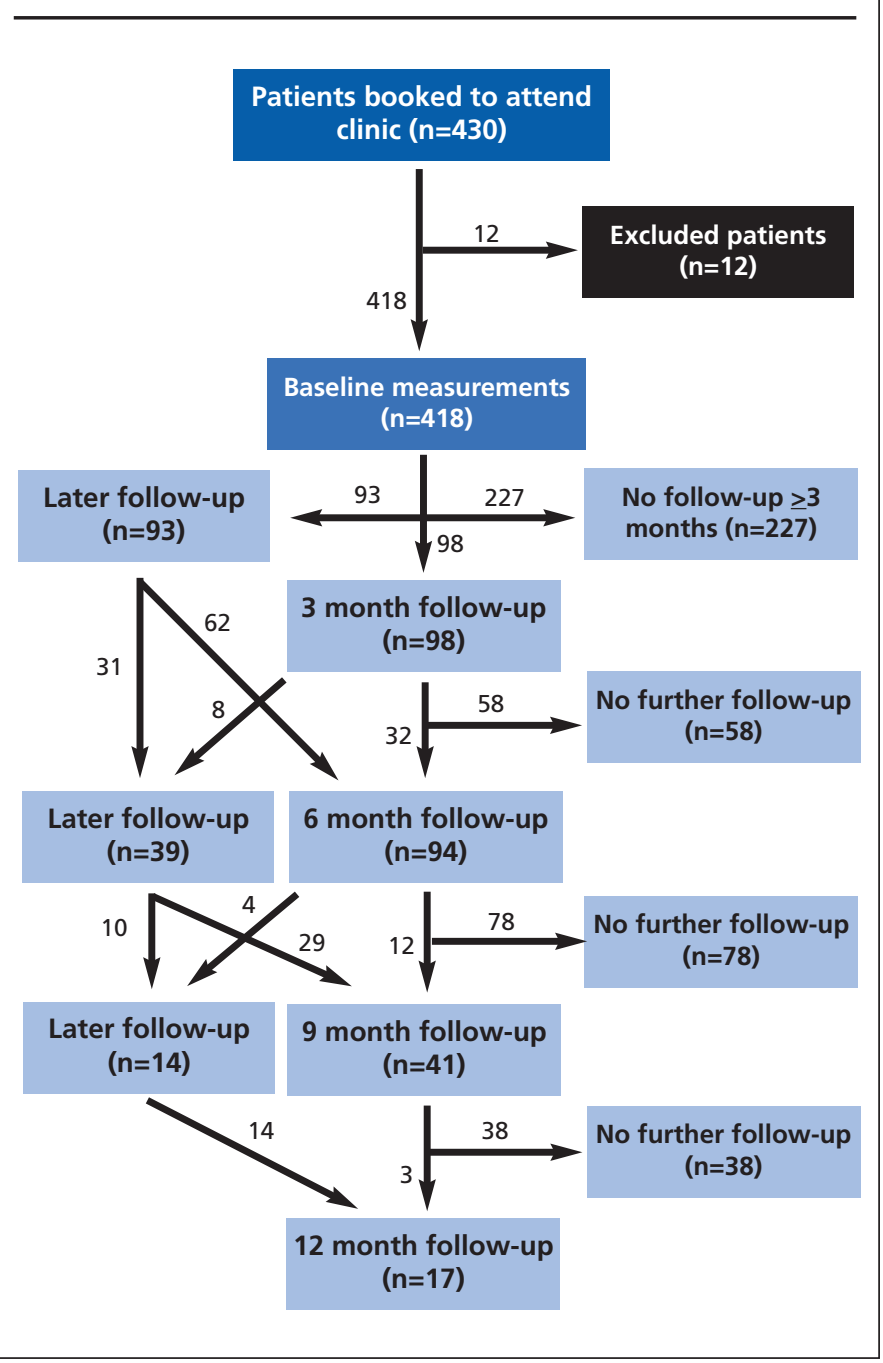

Commonly occurring co-morbidities included type 2 diabetes mellitus (T2DM) (31.1\%), hypertension (31.1\%), depression (26.1\%), obstructive sleep apnoea (23.2\%) and osteoarthritis (15.6\%). Table 2 details the top 30 co-morbidities. When assessing for co-morbidity and weight clustering according to age threshold, no significant differences were noted for the prevalence of co-morbidities according to age thresholds of $<35$ years, 35-49 years, 50-64 years and $>65$ years.

\section{Baseline weight according to age}

The mean \pm SD weight and BMl for patients aged $<35$ years was $143.6 \pm 30.8 \mathrm{~kg}$ and $49.7 \pm 9.3 \mathrm{~kg} / \mathrm{m}^{2}$, respectively. Respective data for those aged $35-49$ years were $137.2 \pm 30.9 \mathrm{~kg}$ and $47.5 \pm 8.5 \mathrm{~kg} / \mathrm{m}^{2}$, for those aged $50-64$ years were $137.0 \pm 26.8$ $\mathrm{kg}$ and $47.9 \pm 8.3 \mathrm{~kg} / \mathrm{m}^{2}$ and for patients aged $>65$ years were $130.2 \pm 25.8 \mathrm{~kg}$ and $47.1 \pm 9.1 \mathrm{~kg} / \mathrm{m}^{2}$.

\section{Weight outcomes}

As a surrogate marker of our patient population and complex
Table 1 Prevalence of various baseline characteristics in the sample

\begin{tabular}{|c|c|c|c|c|}
\hline Characteristic & Number & Mean/\% & SD & Range \\
\hline Age & 418 & 47 & 12 & 59 \\
\hline Male & 122 & $29.2 \%$ & & \\
\hline Female & 296 & $70.8 \%$ & & \\
\hline Baseline weight $(\mathrm{kg})$ & 418 & 137.2 & 29.2 & 185.6 \\
\hline Body mass index $\left(\mathrm{kg} / \mathrm{m}^{2}\right)$ & 418 & 48.0 & 8.6 & 56.9 \\
\hline White British & 267 & $63.9 \%$ & & \\
\hline Unknown/not stated & 115 & $27.5 \%$ & & \\
\hline White Other & 13 & $3.1 \%$ & & \\
\hline Indian & 3 & $0.7 \%$ & & \\
\hline Pakistani & 3 & $0.7 \%$ & & \\
\hline Black Caribbean & 3 & $0.7 \%$ & & \\
\hline Black African & 2 & $0.5 \%$ & & \\
\hline White/Black Caribbean & 2 & $0.5 \%$ & & \\
\hline Mixed Other & 2 & $0.5 \%$ & & \\
\hline Other & 2 & $0.5 \%$ & & \\
\hline White and Asian & 2 & $0.5 \%$ & & \\
\hline Asian Other & 1 & $0.2 \%$ & & \\
\hline Bangladeshi & 1 & $0.2 \%$ & & \\
\hline White Irish & 1 & $0.2 \%$ & & \\
\hline White/Black African & 1 & $0.2 \%$ & & \\
\hline Overweight & 4 & $1.0 \%$ & & \\
\hline Class I Obese & 12 & $2.9 \%$ & & \\
\hline Class II Obese & 48 & $11.5 \%$ & & \\
\hline Class III Obese & 354 & $84.7 \%$ & & \\
\hline
\end{tabular}

needs at presentation, in order to allow comparison with other evaluation studies we undertook assessment of short-term weight outcomes of our cohort. A significant reduction in median weight was observed at 3 months $(135.9 \mathrm{~kg}$ vs. 134.4 $\mathrm{kg} ; \mathrm{p}<0.001)$ and 6 months (135.8 kg vs. $130.8 \mathrm{~kg} ; \mathrm{p}<0.001)$. However, non-significant weight loss was observed at 9 months, possibly due to the smaller number of patients at follow-up as the majority of patients would have been transferred to Tier 4 service at this stage of follow-up (136.4 kg vs. $128 \mathrm{~kg}$; $\mathrm{p}=0.116)$. Overall, 43 patients out of a total of 191 (22.5\%) with followup data achieved weight loss of $\geq 5 \%$ of their baseline weight. This equates to a success rate of $22.5 \%$. Similar patterns of weight loss were observed for males and females - that is, significant weight loss at 3 and 6 months, non-significant weight loss at 9 months (Table 3).

\section{Discussion}

This evaluation highlights the challenges of tackling complex obesity and related co-morbidities. A significant number of patients presenting to our hospital-based Tier 3 service had significant co-morbidities at baseline and would therefore require complex medical, dietetic and psychological intervention prior to bariatric surgery. Some of the baseline characteristics for 
Table 2 Prevalence of the 30 most common diseases in the sample

\begin{tabular}{lll}
\hline Disease & Number & $\%$ \\
\hline Type 2 diabetes mellitus & 130 & 31.1 \\
Hypertension & 130 & 31.1 \\
Depression & 109 & 26.1 \\
Obstructive sleep apnoea & 97 & 23.2 \\
Osteoarthritis & 65 & 15.6 \\
Asthma & 62 & 14.8 \\
Limited mobility & 60 & 14.4 \\
Gastroesophageal reflux disease & 43 & 10.3 \\
Chronic back pain & 41 & 9.8 \\
Polycystic ovarian syndrome & 38 & 9.1 \\
Anxiety & 38 & 9.1 \\
Fibromyalgia & 32 & 7.7 \\
Hypothyroidism & 32 & 7.7 \\
Non-alcoholic fatty liver disease & 21 & 5.0 \\
Lymphoedema & 19 & 4.5 \\
Arthritis (not specified) & 18 & 4.3 \\
Coronary heart disease & 15 & 3.6 \\
Hiatus hernia & 15 & 3.6 \\
Irritable bowel syndrome & 15 & 3.6 \\
High cholesterol & 12 & 2.9 \\
Chronic knee pain & 12 & 2.9 \\
Vitamin D deficiency & 11 & 2.6 \\
Heartburn & 10 & 2.4 \\
Atrial fibrillation & 10 & 2.4 \\
Chronic obstructive pulmonary disease & 9 & 2.2 \\
Migraine & 9 & 2.2 \\
Diabetic retinopathy & 9 & 2.2 \\
Previous myocardial infarction & 9 & 2.2 \\
Peripheral neuropathy & 8 & 1.9 \\
Previous cerebrovascular accident & 7 & 1.7 \\
& & \\
& & \\
\hline
\end{tabular}

Table 3 Change in median baseline and follow-up weights at all the stages of follow-up with Wilcoxon signed rank test results and $p$ values

\begin{tabular}{llll}
\hline & 3 months & $\mathbf{6}$ months & $\mathbf{9}$ months \\
Patients with weight recorded & 98 & 94 & 41 \\
Median baseline weight $(\mathrm{kg})$ & 135.9 & 135.8 & 136.4 \\
Median follow-up weight $(\mathrm{kg})$ & 134.2 & 130.8 & 128.0 \\
Wilcoxon signed rank test result & 1036 & 799.5 & 277.5 \\
P value & $<0.001$ & $<0.001$ & 0.116
\end{tabular}

our cohort were similar to other studies. For example, $70.8 \%$ of our cohort were female compared with $70.0 \%$ in the study by Jennings et al. ${ }^{11}$ The high proportion of female patients attending a specialist weight management programme is well described. The major co-morbidities in our sample closely matched those in the study by Jennings et al: type 2 diabetes (31.1\% vs. $31.7 \%$ ), hypertension (31.1\% vs. $38.3 \%$ ), depression (26.1\% vs. 31.3\%). However, there were also a few differences, notably, the presence of sleep apnoea (23.2\% vs. $11.7 \%)$ and coronary heart disease (3.6\% vs. 11.7\%). These discrepancies are likely because our clinical service was specifically designed to screen for co-morbidities, which therefore included detailed tests and questionnaire assessments at baseline.

Despite the challenges of treating complex obesity and despite the fact that this was not the main remit of this evaluation study, we undertook assessment of short-term weight outcome in order to allow comparison with other evaluation studies with regard to differences in patient population and their complex needs. We observed significant weight loss at 3 and 6 months; $22.5 \%$ of patients achieved $\geq 5 \%$ weight loss from baseline, which is relevant because $5 \%$ loss of baseline weight has been associated with an array of metabolic improvements, most notably improvements in systemic insulin sensitivity, beta cell function and cardio-metabolic parameters. $9,1322.5 \%$ of patients with any length of follow-up lost $\geq 5 \%$ of their baseline weight during their course of treatment. This value is consistent with the $23.7 \%$ of patients who lost $\geq 5 \%$ of their baseline weight at 6 12 months, excluding patients with a follow-up of 3 months or no follow-up at all. In comparison with other evaluation studies, our weight outcomes were comparable to a report from another specialist weight management programme in Glasgow, UK where $24 \%$ of patients experienced a $\geq 5 \%$ weight loss at 12 months of the intervention period. ${ }^{14}$ However, our findings compare negatively with other similar schemes elsewhere. Jennings et $a^{111}$ reported a value of $60 \%$ for their entire cohort whereas another study from Canada reported a value of $47 \%$ at 12 months. ${ }^{15}$ Crucially, the baseline BMl for our patient cohort (48 $\mathrm{kg} / \mathrm{m}^{2}$ ) was higher than that from these other cohorts (44.1 $\mathrm{kg} / \mathrm{m}^{2}, 43.3 \mathrm{~kg} / \mathrm{m}^{2}$ and $44.7 \mathrm{~kg} / \mathrm{m}^{2}$, respectively), ${ }^{11,14,15}$ suggesting that patients from our cohort had more severe obesity and related co-morbidities and were likely to require more complex medical, dietetic and psychological interventions. Our patient cohort is also significantly different from commercial weight loss programmes; for example, Weight Watchers ${ }^{\mathrm{TM}}$ reported $51 \%$ of participants losing $5 \%$ of body weight with a mean BMI at baseline of $38 \mathrm{~kg} / \mathrm{m}^{2},{ }^{16}$ while the Lighten Up study used patient selfreported final weight as an outcome measure, ${ }^{17}$ which is known to be inaccurate due to underreporting of weight. ${ }^{18}$

The ethnic demographics of the sample were largely similar to the 2011 census data for the East Midlands. ${ }^{19}$ Although White British was reported as $63.9 \%$, the ethnicity of $27.5 \%$ of the sample was unknown or not stated, so it is likely that the percentage of White British is within a few percent of the census figure of $85.4 \%$ if the $27.5 \%$ is distributed in our sample. White Other was reported as 3.1\% in our sample compared with 3.2\% in the census. The collective South-East Asian ethnicities of our sample totalled $1.8 \%$, which appears lower than the census figure of $5.6 \%$; however, the unknown and not stated ethnicity of our series may account for this. 


\section{Key messages}

- There is limited evidence on the prevalence of patients' obesity-related co-morbidities and the effectiveness of specialist multidisciplinary weight management (Tier 3) programmes prior to bariatric surgery

- The most common co-morbidities recorded at baseline were type 2 diabetes mellitus (31.1\%), hypertension (31.1\%), depression (26.1\%), obstructive sleep apnoea (23.2\%) and osteoarthritis (15.6\%)

- Significant weight loss was observed at the 3-month and 6-month follow-up points, but not at the 9- or 12-month follow-up points. $22.5 \%$ of patients achieved weight loss of $\geq 5 \%$

Our drop-out rate at 12 months was $12.2 \%$ for 2017 , which compares positively with drop-out rates from three other studies of $14.3 \%, 62.5 \%$ and $51.0 \%$, respectively. $11,20,21$ This was reassuring since our centre accepted patients from a wide geographical area (serving a population of over 3 million inhabitants and a geographic area of 4500 square miles), which may affect the practicality of travelling for patients' regular follow-up appointments. In addition to the practicalities of attending follow-up visits, some patients may choose to depart the service for reasons other than poor motivation; for example, patients may choose not to progress to surgery and were discharged as a result.

Some weaknesses of our study should be highlighted. Whilst the baseline information recorded for patients was comprehensive, some measure of education status and socioeconomic status would be desirable. A measure of waist circumference when practical would also be ideal as it serves as a measure of central body fat, which is a risk factor for coronary heart disease and type 2 diabetes and is recommended by Public Health England and $\mathrm{NICE}, 22,23$ but, practically, this is not feasible in patients who are severely obese. Follow-up sessions currently lack consistent retesting of co-morbid biochemical parameters such as $\mathrm{HbA}_{1 \mathrm{c}}$, lipid and liver function profile as well as objective assessments of respiratory, cardiovascular and musculoskeletal parameters. Follow-up quality of life and anxiety questionnaires (PHQ-9 and GAD-7) would have allowed us the ability to quantitatively assess the psychological presentation and change of our patients. Additional questionnaires could also encompass baseline physical activity as well as fruit and vegetable consumption.

In conclusion, the Tier 3 service provided by EMBMI was fully compliant with relevant commissioning guidelines. The high prevalence of co-morbidities highlighted in this study provide a basis for future studies to move away from a weight-centric view of assessing effectiveness of a specialist weight management programme and to focus on improvement or resolution of base- line co-morbidities as an important primary outcome measure. Indeed, an important remit of our service is to ensure optimal management of co-morbidities prior to surgery, either via our Tier multidisciplinary management or by referral to specialist colleagues. Assessment of our patients' baseline weight and weight outcomes also provide a surrogate of a measure of the complex need for our patients presenting to a hospital-based Tier 3 programme compared with previous studies. In addition, our study supports the need for a standardised Tier 3 assessment process which includes standardised outcome measures for obesityrelated co-morbidities.

\section{Conflict of interest None declared. \\ Funding None.}

Acknowledgement This work was supported by the Medical Research Council (grant numbers MR/K00414X/1, MR/P021220/1) and Arthritis Research UK (grant number 19891).

\section{References}

1. World Health Organization. Controlling the Global Obesity Epidemic. 2017. Available at: http://www.who.int/nutrition/topics/obesity/en/ (accessed 4 Dec 2017).

2. NHS Digital. Statistics on Obesity, Physical Activity and Diet. 2017. Available at: https://www.gov.uk/government/uploads/system/uploads/attachment_data/file/613532/obes-phys-acti-diet-eng-2017-rep.pdf.

3. Field $A E$, Byers $T$, Hunter DJ, et al. Weight cycling, weight gain, and risk of hypertension in women. Am J Epidemiol 1999;150:573-9. https://doi.org/ 10.1093/oxfordjournals.aje.a010055

4. Maclure KM, Hayes KC, Colditz GA, et al. Weight, diet, and the risk of symptomatic gallstones in middle-aged women. N Engl J Med 1989; 321:563-9. https://doi.org/10.1056/NEJM198908313210902

5. Hartz AJ, Fischer ME, Bril G, et al. The association of obesity with joint pain and osteoarthritis in the HANES data. J Chronic Dis 1986;39:311-19. https://doi.org/10.1016/0021-9681(86)90053-6

6. Hubert HB, Feinleib M, McNamara PM, Castelli WP. Obesity as an independent risk factor for cardiovascular disease: a 26-year follow-up of participants in the Framingham Heart Study. Circulation 1983;67:968-77. https://doi.org/10.1161/01.CIR.67.5.968

7. Vgontzas AN, Tan TL, Bixler EO, Martin LF, Shubert D, Kales A. Sleep apnea and sleep disruption in obese patients. Arch Intern Med 1994;154:170511. https://doi.org/10.1001/archinte.1994.00420150073007

8. Adler M, Schaffner F. Fatty liver hepatitis and cirrhosis in obese patients. Am J Med 1979;67:811-16. https://doi.org/10.1016/0002-9343(79)90740-X

9. Wing RR, Lang W, Wadden TA, et al. Benefits of modest weight loss in improving cardiovascular risk factors in overweight and obese individuals with type 2 diabetes. Diabetes Care 2011;34:1481-6. https://doi.org/10.2337/ dc10-2415

10. NHS Commissioning Board Clinical Reference Group for Severe and Complex Obesity. Clinical Commissioning Policy: Complex and Specialised Obesity Surgery. 2013. Available at: https://www.england.nhs.uk/wp-content/uploads/2016/05/appndx-6-policy-sev-comp-obesity-pdf.pdf

11. Jennings $A$, Hughes $C A$, Kumaravel $B$, et al. Evaluation of a multidisciplinary Tier 3 weight management service for adults with morbid obesity, or obesity and comorbidities, based in primary care. Clin Obes 2014;4:254-66. https://doi.org/10.1111/cob.12066

12. Public Health England. Standard Evaluation Framework for Weight Management Interventions. London, 2018. Available at: https://assets.publishing.service.gov.uk/government/uploads/system/uploads/attachment_data/fil e/685545/SEF_weight_management_interventions.pdf

13. Magkos F, Fraterrigo G, Yoshino J, et al. Effects of moderate and subsequent progressive weight loss on metabolic function and adipose tissue biology in humans with obesity. Cell Metab 2016;23:591-601. https://doi.org/ 10.1016/j.cmet.2016.02.005.

14. Logue J, Allardice G, Gillies M, Forde L, Morrison DS. Outcomes of a specialist weight management programme in the UK National Health Service: 
prospective study of 1838 patients. BMJ Open 2014;4:e003747. https://doi.org/10.1136/bmjopen-2013-003747

15. Kamga-Ngandé CN, Carpentier AC, Nadeau-Marcotte F, et al. Effectiveness of a multidisciplinary program for management of obesity: the Unité d'Enseignement, de Traitement et de Recherche sur l'Obésité (UETRO) database study. Metab Syndr Relat Disord 2009;7:297-304. https://doi.org/ 10.1089/met.2008.0063

16. Dixon KJ, Shcherba S, Kipping RR. Weight loss from three commercial providers of NHS primary care slimming on referral in North Somerset: service evaluation. J Public Health (Oxf) 2012;34:555-6. https://doi.org/ 10.1093/pubmed/fds034

17. Jolly K, Lewis A, Beach J, et al. Comparison of range of commercial or primary care led weight reduction programmes with minimal intervention control for weight loss in obesity: Lighten Up randomised controlled trial. BMJ 2011;343:d6500. https://doi.org/10.1136/bmj.d6500

18. Gorber CS, Tremblay M, Moher D, Gorber B. A comparison of direct vs. self-report measures for assessing height, weight and body mass index: a systematic review. Obes Rev 2007;8:307-26. https://doi.org/10.1111/ j.1467-789X.2007.00347.x
19. Office for National Statistics. Ethnic Groups - East Midlands. London. 2012. Available at: https://www.ons.gov.uk/peoplepopulationandcommunity/ populationandmigration/populationestimates/datasets/censusoutputare aestimatesintheeastmidlandsregionofengland

20. Morrison DS, Boyle S, Morrison C, Allardice G, Greenlaw N, Forde L. Evaluation of the first phase of a specialist weight management programme in the UK National Health Service: prospective cohort study. Public Health Nutr 2012;15:28-38. https://doi.org/10.1017/S1368980011001625

21. Senior L, Carter D, Capehorn M. Service evaluation of the Rotherham Institute for Obesity and comparison of 2010 and 2011 data. Obes Facts 2013;6(Suppl. 1):116.

22. Public Health England. Adult Obesity and Type 2 Diabetes. London, 2014. Available at: https://assets. publishing.service.gov.uk/government/uploads/ system/uploads/attachment_data/file/338934/Adult_obesity_and_type_2_di abetes_.pdf

23. NICE. Obesity identification, assessment and management. London, 2014. Available at: https://www.nice.org.uk/guidance/cg189

\section{Diabetes Professional Care $^{\circ}-$ DPC $^{\circ}$} \#DPC2019
$29 \& 30$ October 2019

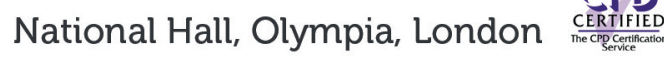

FREE TO ATTEND FOR HCPS

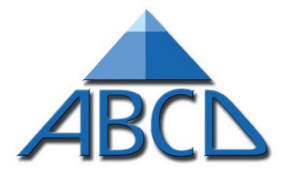

ish Clinical Diabetologiss

\section{Don't miss the latest updates in $A B C D$ Theatre at Diabetes Professional Care (DPC)}

Join $6,000+$ HCPs on 29 \& 30 October for the most broad-reaching, comprehensive programme of real-world education and clinical skills designed for the entire multi-disciplinary team.

As an $A B C D$ member, you will have access to all areas - including nine main conference streams, seven practical clinics, and your very own ABCD Theatre and Lounge.

If you come in to contact with people living with diabetes, this is a must attend event for you!
"It felt like DPC was the perfect fit as it has successfully delivered vital CPD-accredited education for all healthcare professionals in diabetes care. We all have one objective and that's to work together to benefit people with diabetes. I can't think of a better way to do this than to come together at the largest, free-to-attend diabetes conference in the country."

Dr Dinesh Nagi ABCD Chair

To register for your free place online visit www.diabetesprofessionalcare.com/register or call 02380811551

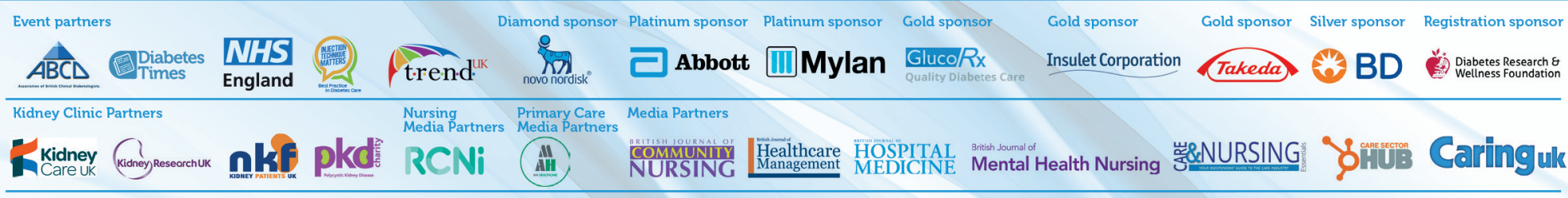

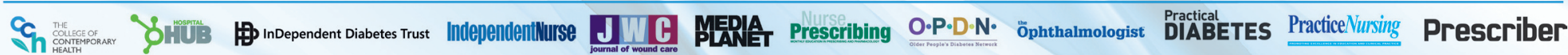

